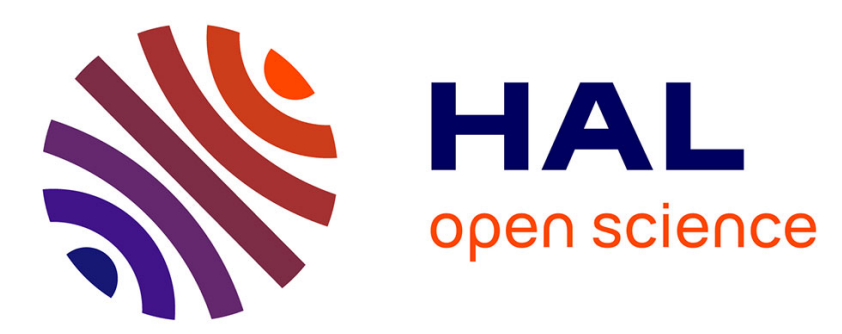

\title{
Evidence for Collective Nonlinear Interactions in X Ray into Ultraviolet Parametric Down-Conversion
}

\author{
D. Borodin, A. Schori, J.-P. Rueff, J. m. M. Ablett, S. Shwartz
}

\section{To cite this version:}

D. Borodin, A. Schori, J.-P. Rueff, J.m. M. Ablett, S. Shwartz. Evidence for Collective Nonlinear Interactions in X Ray into Ultraviolet Parametric Down-Conversion. Physical Review Letters, 2019, 122 (2), pp.023902. 10.1103/PhysRevLett.122.023902 . hal-02313585

\section{HAL Id: hal-02313585 \\ https://hal.sorbonne-universite.fr/hal-02313585}

Submitted on 11 Oct 2019

HAL is a multi-disciplinary open access archive for the deposit and dissemination of scientific research documents, whether they are published or not. The documents may come from teaching and research institutions in France or abroad, or from public or private research centers.
L'archive ouverte pluridisciplinaire HAL, est destinée au dépôt et à la diffusion de documents scientifiques de niveau recherche, publiés ou non, émanant des établissements d'enseignement et de recherche français ou étrangers, des laboratoires publics ou privés. 


\title{
Evidence for collective nonlinear interactions in $\mathrm{x}$ ray into ultraviolet parametric down-conversion
}

\author{
D. Borodin ${ }^{1}$, A. Schori ${ }^{1}$, J.-P. Rueff ${ }^{2,3}$, J. Ablett $^{2}$, and S. Shwartz ${ }^{1^{*}}$ \\ ${ }^{1}$ Physics Department and Institute of Nanotechnology, Bar-Ilan University, Ramat Gan, \\ 52900 Israel \\ ${ }^{2}$ Synchrotron SOLEIL L'Orme des Merisiers, BP 48 Saint-Aubin, 91192 Gif-sur-Yvette, \\ France \\ ${ }^{3}$ Sorbonne Université, CNRS, Laboratoire de Chimie Physique-Matière et \\ Rayonnement, LCPMR, F-75005 Paris, France
}

\begin{abstract}
We present the observation of peculiar non-monotonic photon energy dependencies of the count rates and of the rocking curves of parametric down-conversion of $\mathrm{x}$ rays into ultraviolet far from any atomic resonances. The observations cannot be explained by models that consider only atomic or bond charge responses and suggest that collective phenomena contribute to the effect. We propose an interpretation that includes nonlinear interactions with plasmons, which can explain the existence of peaks in this energy range. Our work implies that nonlinear interactions between $\mathrm{x}$ rays and either ultraviolet or visible radiation can be utilized as a powerful atomic scale probe for collective effects in solids.
\end{abstract}

\section{*sharon.shwartz@biu.ac.il}


The field of nonlinear interactions between $\mathrm{x}$ rays and longer wavelengths has made a major progress in recent years [1-9]. These processes are interesting, since they present novel physics involving fundamental aspects of light-matter interactions arising from the large diversity of the involved wavelengths, and since they can be used to probe phenomena related to valence electrons at the atomic scale resolution [9, 10]. This is because the long optical wavelengths interact with the valence electrons and the $\mathrm{x}$ rays provide the high spatial resolution. This effect can be viewed as x-ray scattering from the optically induced valence charge variations. The photon energy of the scattered $\mathrm{x}$ rays is shifted by the amount that is equal to the photon energy of the longer wavelength wave to conserve energy. In a typical experiment, the requirement for momentum conservation (phase matching) is fulfilled by using one of the reciprocal lattice vectors of the nonlinear crystal. Consequently, the efficiency of the effect is propositional to the absolute square of the Fourier component of the induced valence charge density corresponding to the selected reciprocal lattice vector. A series of measurements of these coefficients can be used for the reconstruction of the microscopic optical response of the valence charges of materials [3].

Most of the valence electronic resonances of materials are in the ultraviolet (UV) range. Since materials are opaque at those wavelengths, nonlinear interactions between $\mathrm{x}$ rays and longer wavelengths have been observed by using the effect of $x$ ray into UV parametric down-conversion (PDC) $[1-3,6-8,11]$. This is a second-order nonlinear process in which a pump photon interacts with the vacuum field in a nonlinear medium, which in turn leads to the generation of a photon pair, where one photon is an x-ray photon and the other is a UV photon. The convention is to denote the x-ray photon as the signal and the UV photon as the idler. The sum of the photon energies of the generated photons is equal to the photon energy of the pump photon. Since the photon pairs are generated simultaneously at the same position, the count rates of the emerging x-ray photons depend on the generation rate of the UV photons. Consequently, although the UV photons are absorbed in the material, the information on phenomena that are associated with energies that correspond to UV wavelengths is retrieved by measuring the $\mathrm{x}$-ray photons. The measurements of the $\mathrm{x}$-ray photons also provide the atomic scale resolution, which is an inherent property of $\mathrm{x}$-rays arising from their short wavelengths. Hence, PDC of x-rays into UV can be used as a probe for the microscopic optical response at extremely high resolution as has been demonstrated by Tamasaku and his colleagues [3]. 
However, the authors of all the previous papers on $\mathrm{x}$ ray and either UV or optical nonlinear interactions have considered only local responses [1-11]. The interactions have been viewed as inelastic $\mathrm{x}$-ray scattering from local charge excitations where both atomic and bond charge responses have been considered [1-7]. In some of the papers the results have been interpreted as Fano interference between the PDC and Compton scattering [1-3].

In this Letter, we extend the study on nonlinear x-ray optics to include the interactions with plasmons. We explore the effect of PDC of $x$ rays into $U V$ in a diamond crystal by measuring the $x$-ray signal that corresponds to idler photons in the energy range of $28-54 \mathrm{eV}$ where our model predicts peaks in the frequency dependence of the efficiency of the effect far from any atomic resonances (the binding energies of electrons in carbon are 12, 16, and $280 \mathrm{eV}$ ). We measure the non-monotonic dependencies of the count rate and of the angular peak positions of the rocking curves on the signal photon energy, which include the predicted peaks but also show more unexplained structures.

The photons that are generated via the PDC process obey energy and momentum conservation (phase matching). The energy conservation equation can be written in the form of $\mathrm{h} \omega_{p}=\mathrm{h} \omega_{s}+\mathrm{h} \omega_{i}$, where $p, s$, and $i$ refer to the pump, the signal, and the idler respectively. Since the x-ray wavelengths are comparable to the interatomic distances, phase matching is achieved by using the reciprocal lattice vector $\stackrel{\boldsymbol{G}}{\boldsymbol{G}}$ [12]. The k-vectors of the pump, signal, and idler waves satisfy the phase-matching equation $\stackrel{1}{k}_{p}+\stackrel{1}{G}=\stackrel{1}{k}_{s}+\stackrel{1}{k}_{i}$, where $k$ denotes the wave vector of the corresponding photon.

We first describe a simple theory that includes the interactions with plasmons and that predicts the existence of peaks far from atomic resonances. We consider a classical model for the nonlinearity that includes excitations of plasmons in the nonlinear medium. The nonlinear signal can be calculated by solving the wave equation with the nonlinear current density as the source term. Consequently, the efficiency of the PDC process is proportional to the absolute square of the nonlinear current density and the information that is encoded in the nonlinear current density can be reconstructed from the measurement of the efficiency of the PDC.

The nonlinear current density can be found as a second-order perturbation of electron motion [13]: 


$$
\stackrel{1}{J}_{s}^{N L}=\rho_{0} v_{s}^{(2)}+\rho_{p}^{(1)} v_{i}^{\mathbf{r}_{(1) *}}+\rho_{i}^{(1) *} v_{p}^{(1)}
$$

where $\rho_{0}$ is the unperturbed charged density, $\rho_{j}^{(1)}$ is the charge density modulated by the $j$-th frequency, and $v_{j}^{(n)}$ is the $n$-th order velocity at the $j$-th frequency. The model consists the equation of motion of an electron with the restoring force and the equation of continuity for the signal and idler waves $[4,14]$ :

$$
\begin{aligned}
& \frac{\partial v_{j}}{\partial t}+\left(v_{j}^{\mathrm{r}} \cdot \nabla\right) \underset{v_{j}}{\mathrm{r}}+\omega_{0}^{2} \stackrel{\mathrm{r}}{r_{j}}=-\frac{q_{e}}{m_{e}}\left(\stackrel{\mathrm{r}}{E_{j}}+\stackrel{\mathrm{r}}{v_{j}} \times \stackrel{\mathrm{r}}{B_{j}}\right) \\
& \frac{\partial \rho_{j}}{\partial t}+\nabla \cdot\left(\rho_{j}{ }_{v_{j}}^{\mathrm{r}}\right)=0
\end{aligned}
$$

where $m_{e}$ is the effective electron mass, $q_{e}$ is the electron electric charge, $B_{j}$ is the magnetic field, $E_{j}$ is the electric field strength of the corresponding wave, and $\omega_{0}$ denotes the resonance frequency. We assume that the motions of electrons at the signal and pump frequencies are free, since their photon energies are well above any electronic binding energy in the nonlinear medium. At the idler frequency we include the restoring force term, since its corresponding photon energy is on the order of the binding energies of the valence electrons. We solve the system of Eq. 2 together with the Maxwell equations [15]. Note, that we take the Gauss's law in the form $\nabla \cdot \stackrel{\mathrm{r}}{E}=\frac{\rho_{j}^{(1)}}{\varepsilon_{0}}$ as in Ref. 14. Our calculations result in the following expression for the envelope of the nonlinear current density

$$
J_{s}^{N L}=\frac{\varepsilon_{0} q_{e} \chi_{G}\left(\omega_{i}\right) E_{i}^{*} E_{p}}{m^{2} \omega_{p}}\left(-\frac{\left(G \cdot \hat{e}_{i}\right)\left(\hat{e}_{p} \cdot \hat{e}_{s}\right)}{\left(1+\frac{\omega_{\text {plasma }}^{2}}{\left(\omega_{o}^{2}-\omega_{i}^{2}\right)}\right)}\right),
$$

where $\chi_{G}^{(1)}\left(\omega_{i}\right)$ is the Fourier component of the linear electric susceptibility that correspond to the reciprocal lattice vector $\stackrel{\mathfrak{G}}{G}$ at the idler frequency, and $\hat{e}$ is the polarization vector of the corresponding wave. We estimate $\chi_{G}^{(1)}\left(\omega_{i}\right)$ for idler energies below $35 \mathrm{eV}$ from the experimental data [16] and for higher idler energies we use the values calculated by Henke et al. [17]. The plasma resonance h $\omega_{\text {plasma }}$ is calculated by 
using the well-known expression for the plasma frequency $\omega_{\text {plasma }}=\sqrt{\frac{q_{e} \rho_{0}}{m_{e} \varepsilon_{0}}}$, and equals 31 $\mathrm{eV}$ for diamond. In our calculations we use the plasma resonance energy of $34 \mathrm{eV}$ due to the dispersion of plasmons in the [110] direction [18]. The expression in Eq. 3 predicts a nonlinear resonance at a frequency, which is equal to $\omega_{i}=\omega_{N L}=\sqrt{\omega_{\text {plasma }}^{2}+\omega_{0}^{2}}$. In Ref. 4 the authors took $\omega_{0}$ to be equal to the band transition of a diamond crystal for the correction of the nonlinear current density. Since in real crystals there is more than one resonance associated with band transitions, we use the resultant nonlinear current density in the following form

$$
J_{t o t}^{N L}=W_{1} J_{s 1}^{N L}+W_{2} J_{s 2}^{N L}+\ldots
$$

where $W_{j}$ are weight coefficients and $J_{s j}^{N L}$ are the nonlinear current densities from Eq. 3 that corresponds to the $j$-th resonance [19]. Weight coefficients indicate the contribution of the corresponding resonance to the resultant process. In the current calculation the best fit with the experimental result is obtained when the weight coefficients are equal. The reason for this is still an open question that should be addressed with a more comprehensive theory.

Figure 1 shows calculations of the spectrum of the resultant nonlinear current density calculated by using Eq. 4 and include resonances at $5.5 \mathrm{eV}, 7.4 \mathrm{eV}, 12 \mathrm{eV}, 14.4$ $\mathrm{eV}, 16 \mathrm{eV}, 20 \mathrm{eV}$, and $24 \mathrm{eV}$, which are associated with band transitions in a diamond crystal as described in the literature [20,21]. The simulation shows that the PDC signal depends strongly on the photon energy of the idler when it is near $\omega_{N L}$. Each spectral feature represents nonlinear interactions between the band transitions and bulk plasmons [19]. This dependence cannot be explained by any theory that does not include non-local interactions. We note that a recent work by Lucchini and colleagues the measurements of absorption on a polycrystalline diamond sample shows the absence of resonant transitions in this photon energy range [22]. The arrows point at the spectral regions where the peaks were experimentally observed. The discussion on that will be provided later in the text.

The experiment was conducted on the GALAXIES beamline at the SOLEIL synchrotron facility [23, 24]. The experimental setup is shown in Fig. 2. We use a monochromatic beam of $9.995 \mathrm{keV}$ using the $\mathrm{Si}(111)$ reflection of the beamline double crystal monochromator. We use the reciprocal lattice vector normal to the $\mathrm{C}(220)$ atomic 
planes of a diamond crystal for the phase matching in reflection (Bragg) geometry. We select the photon energy of the signal by using the beamline Rowland circle RIXS spectrometer. The instrument consist of a spherically bent $\operatorname{Si}(555)$ crystal analyzer with a curvature radius of one meter and a hybrid-type pixelated 2D MERLIN detector. We estimate that the photon energy resolution of the receiving optics is about $0.5 \mathrm{eV}$. We use an adjustable iris in front of the analyzer to reduce the background radiation along with spatial filtering of the detector image. Typical iris diameter during the measurements was about $5 \mathrm{~mm}$.

The first experimental step is to verify that we observe PDC by confirming that the phase-matching conditions are satisfied. We measure the rocking curves of the x-ray signal that correspond to various idler photon energies as we show in Fig. 3. The peak positions of the rocking curves are slightly shifted from the solutions of the kinematic phasematching equation as we observe also in the simulations. This is because the rocking curves include a large number of the vacuum fluctuation modes with different weights that are determined by the strength of the nonlinearity and the boundary conditions at the various modes as discussed in Ref. 7. The inset in Fig. 3 shows the comparison between the experimentally observed peaks and that obtained in the simulations. The difference between the estimated offset from Bragg and measured is about $0.01 \mathrm{deg}$. We note that the difference between the calculated and measured shifts is smaller than the width of the curves (about 0.02-0.03 deg). The phase mismatch $\Delta k_{z} L_{a b s}$, where $L_{a b s}$ is the absorption length, at all measured peaks of the PDC rocking curves is about 0.1 , which is much smaller than $2 \pi$. As we expect, the peak position shifts farther apart from the Bragg angle as the idler photon energy increases. These are clear indications that the rocking curves in Fig. 3 constitute conclusive evidence that we observe PDC.

Next, we explore the dependencies of the signal count rates and the rocking curve peak positions on the photon energy of the idler and show that as predicted by our theory, the dependencies are not monotonic in the energy range of $28-54 \mathrm{eV}$, which is far from any atomic resonances. For this purpose we measure rocking curves similar to those shown in Fig. 3 for photon energies of the signal in this range in steps of $1 \mathrm{eV}$. In Figs. 4(a) and 4(b) we plot the measured count rates at the peaks of the rocking curves (power spectrum) and relative positions of rocking curve peaks with respect to the angle of elastic scattering as a function of the photon energy of the idler. In the measured power spectrum we see two spectral features above the monotonic decrease of the signal intensity around $36 \mathrm{eV}$ and 
around $42 \mathrm{eV}$ as predicted by the model we described above. The existence of this nonmonotonic dependence of the power spectrum on the idler photon energy, indicates that the underlying physical mechanism for the nonlinear interactions must include origin that is not from atomic interactions since the structures are obtained far from any atomic resonances. According to the theory we have derived above, it can be explained by including interactions with plasmons. A marginal feature around $47 \mathrm{eV}$ is not present in the simulations. This might indicate the contribution of interband transitions in the diamond crystal with higher energies. The corresponding energy of this transition should be around 32-33 eV. Since the feature is very small, the weight coefficients from Eq. (4) corresponding to that transition should be also very small.

While the experimental observed structures of the power spectrum indicate clearly on the nonlinear interactions with the plasmons, the detailed comparison with the theory requires the full simulation of the PDC process since the count rate of the signal depends not just on the nonlinearity but also on the refractive index and the absorption, which are also photon energy dependent. We simulate the PDC process by solving the coupled slowly varying envelope wave equations in the Heisenberg-Langevin model [25] together with the model for the nonlinearity described above. The count rate is calculated by integration the solution for the signal over the photon energies and the solid angle of the experimental detection system. The results of the simulation are shown in the inset of the Fig. 4(a) where the arrows point at the positions of the measured spectral features. We note that most of the features from the calculated nonlinear current density appear in the measured power spectrum. The measured features are wider than predicted by the simulations and some of the spectral features that are predicted by our model are not resolved in the experiment due to the measurement resolution. The simulation also predicts a much higher enhancement of the PDC efficiency near $\omega_{N L}$ than the efficiency that we observe in the experiment. This is probably because of losses that are associated with the plasmon interactions and that we do not consider in our simulation. The selection rules for the nonlinear interactions are also needed to take into account to improve the agreement between the experiment and the theory. In addition, we believe that a comprehensive model that includes a band structure calculation is required for a better description of the effect.

In Fig. 4(b) there is a distinct sharp change in the curve at $45 \mathrm{eV}$, which is also seen in the inset that shows the rocking curves of the signal at the corresponding points. 
Interestingly, the comparison with Fig. 4(a) indicates that at the same photon energy there is a small dip in power spectrum. Such a feature does not appear in the simulation and requires further investigations.

In conclusion, we have observed experimentally non-monotonic photon energy dependencies of PDC of $x$ rays into UV far from any atomic resonances. These results cannot be explained by theories that consider only atomic or bond charge responses. We have proposed and described an explanation for the photon energies dependencies in the measured range by adding interactions with bulk plasmons to the classical model for $x$-ray nonlinearity. While the main idea of strong photon energy dependencies near the nonlinear plasmon is clearly shown in the experimental results, there are still some fine structures in the measured photon energy dependencies that call for a more comprehensive model that includes multiple resonances and the effect of the crystal field. We note that while PDC is a nonlinear effect, its efficiency depends linearly on the intensity of the input beam since it interacts with the vacuum field, which provides the UV fields that is mixed with the input $\mathrm{x}$-ray field. Consequently, PDC experiments can be conducted with storage ring based synchrotrons as we have demonstrated in this work. The experimental results and the theory we described indicate on an unexplored field of nonlinear interactions between $\mathrm{x}$ rays and plasmons and open the possibility for the development of a novel technique that will be used as a probe with atomic-scale resolution for collective excitations in crystals.

This work was supported by the Israel Science Foundation (ISF), grant number 201/17. We acknowledge SOLEIL synchrotron for the provision of synchrotron facilities (Proposal No. 20160046).

\section{References}

[1] K. Tamasaku and T. Ishikawa, Phys. Rev. Lett. 98, 244801 (2007).

[2] K. Tamasaku, K. Sawada, and T. Ishikawa, Phys. Rev. Lett. 103, 254801 (2009).

[3] K. Tamasaku, K. Sawada, E. Nishibori, and T. Ishikawa, Nat. Phys. 7, 705 (2011).

[4] T.E. Glover et. al., Nature 488, 603 (2012). 
[5] E. Shwartz and S. Shwartz, Opt. Express 23, 7471 (2015).

[6] B. Barbiellini, Y. Joly \& K. Tamasaku, Phys. Rev. B 92, 155119 (2015).

[7] D. Borodin, S. Levy, and S. Shwartz, Appl. Phys. Lett. 110, 13 (2017).

[8] A. Schori, C. Bömer, D. Borodin, S. P. Collins, B. Detlefs, M. Moretti Sala, S. Yudovich, and S. Shwartz, Phys. Rev. Lett. 119, 253902 (2017).

[9] J. R. Rouxel, M. Kowalewski, K. Bennett, and S. Mukamel, Phys. Rev. Lett. 120, $243902(2018)$

[10] I. Freund, Chem. Phys. Lett. 12, 583 (1972).

[11] H. Danino and I. Freund, Phys. Rev. Lett., 46, 1127 (1981).

[12] I. Freund, Phys. Rev. Lett. 21, 1404 (1968).

[13] P. M. Eisenberger and S. L. McCall, Phys. Rev. Lett. 26, 684 (1971).

[14] Y. R. Shen, The Principles of Nonlinear Optics (Wiley, 1984).

[15] See Supplemental Material Section 1.

[16] H. R. Phillip and E. A. Taft, Phys. Rev. 136, A1445 (1964).

[17] B. L. Henke, E. M. Gullikson, and J. C. Davis, At. Data Nucl. Data Tables 54, 181 (1993).

[18] S. Waidmann, M. Knupfer, B. Arnold, and J. Fink, Phys. Rev. B 61, 10149 (2000).

[19] See Supplemental Material Section 2.

[20] W. C. Walker and J. Osantowski, Phys. Rev. 134, A153 (1964).

[21] R. Keown, Phys. Rev. 150, 568 (1966).

[22] M. Lucchini, S. A. Sato, A. Ludwig, J. Herrmann, M. Volkov, L. Kasmi, Y. Shinohara, K. Yabana, L. Gallmann, and U. Keller, Science 353, 916 (2016).

[23] J.-P. Rueff, J. M. Ablett, D. Céolin, D. Prieur, T. Moreno, V. Balédent, B. Lassalle-Kaiser, J. E. Rault, M. Simon, and A. Shukla, J. Synchrotron Rad. 22, 175 (2015). 
[24] J. M. Ablett, D. Prieur, D. Céolin, B. Lassalle-Kaiser, B. Lebert, M. Sauvage, T. Moreno, S. Bac, V. Balédent, A. Ovono, M. Morand, F. Gélebart, A. Shukla and J.-P. Rueff, J. Synchrotron Rad. 26, (2019).

[25] S. Shwartz, R. N. Coffee, J. M. Feldkamp, Y. Feng, J. B. Hastings, G. Y. Yin, and S. E. Harris, Phys. Rev. Lett. 109, 013602 (2012).

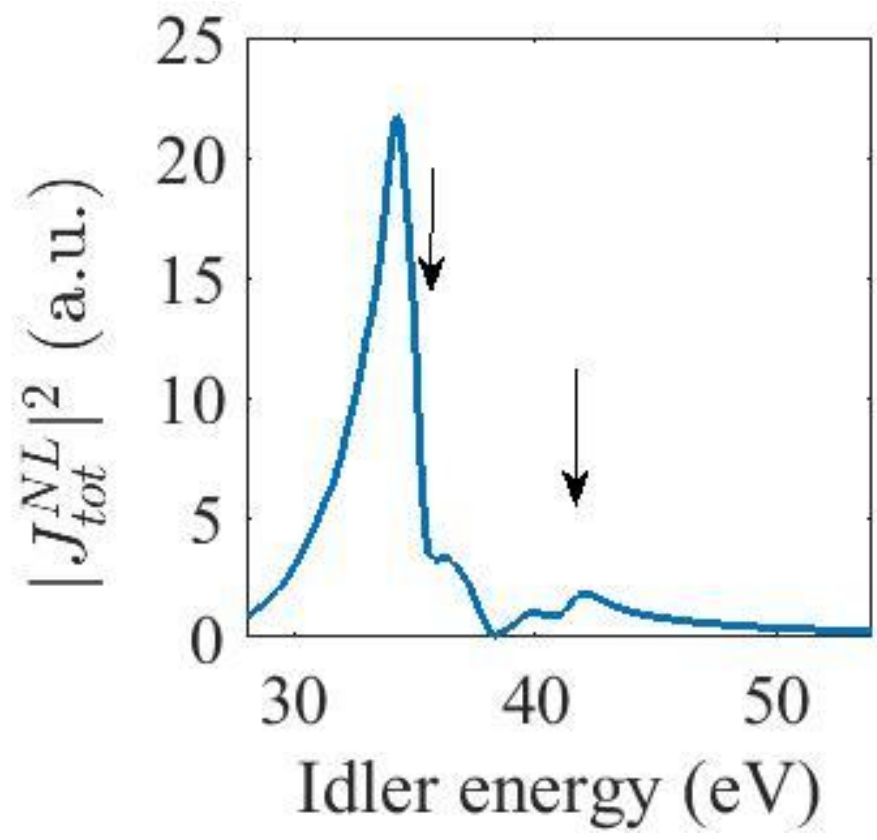

FIG. 1. Calculated nonlinear current density including the interactions with bulk plasmons in diamond. The arrows indicate the spectral regions where we observe the peaks in the experiment. 


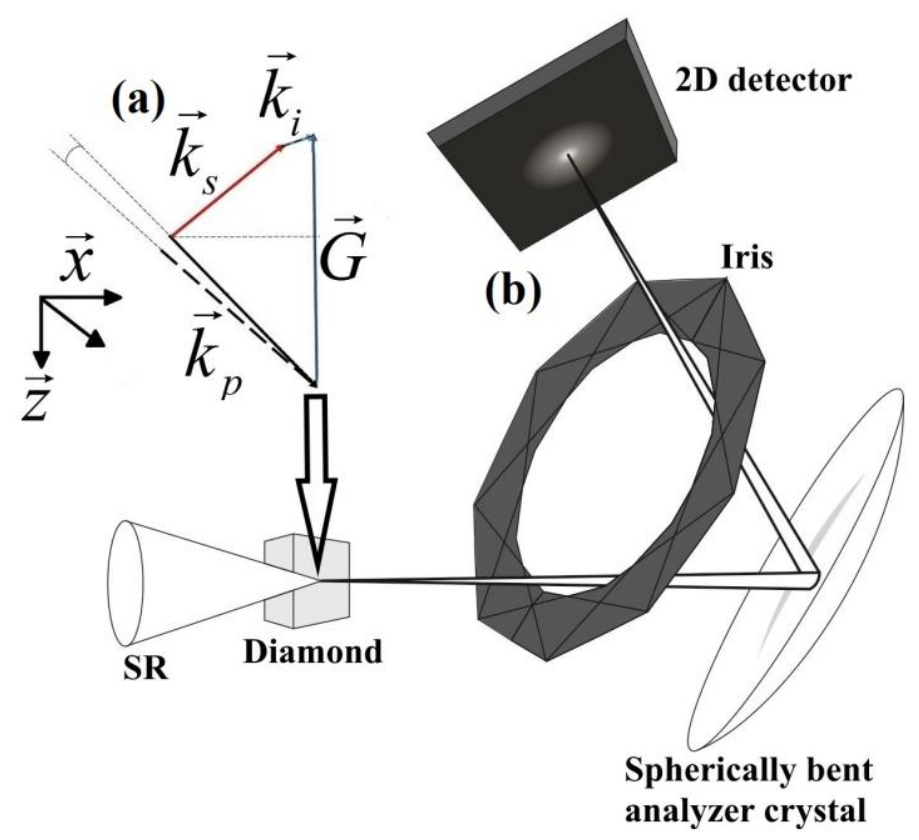

FIG. 2. (Color online) (a) Phase-matching scheme where the indices $p, s$, and $i$ stand for pump, signal, and idler respectively. The dashed line indicates the Bragg diffraction direction. (b) Experimental setup. The synchrotron radiation (SR) illuminates a diamond crystal, which is tuned to an angle satisfying the phase-matching conditions. The PDC signal is collected by a bent crystal analyzer, which images the beam at the selected energy onto a two-dimensional detector. 


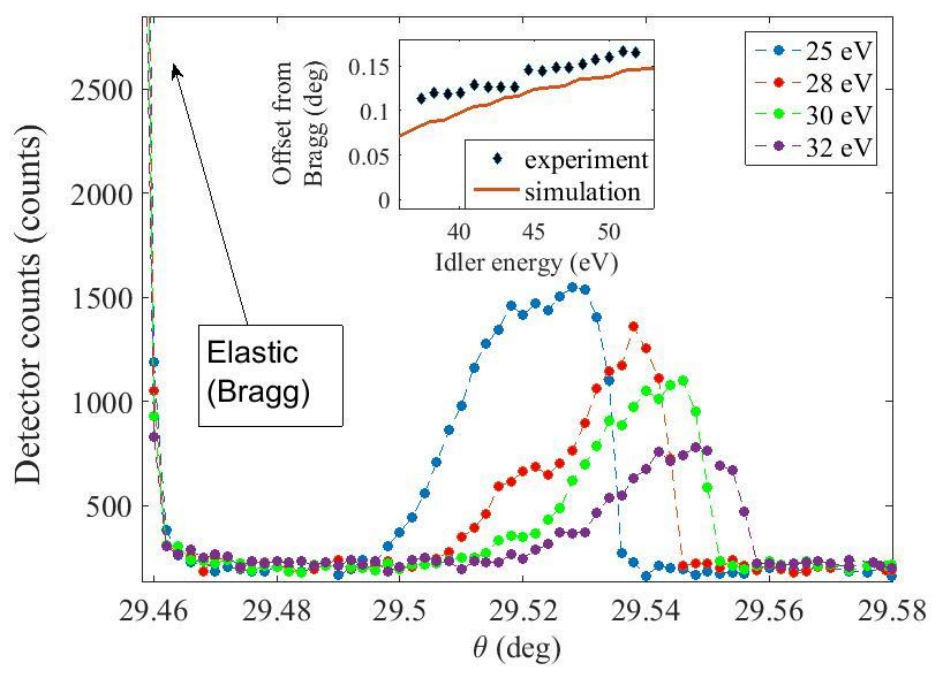

FIG. 3. (Color online) Rocking curves of the PDC signal for corresponding idler photon energies at $25 \mathrm{eV}, 28 \mathrm{eV}, 30 \mathrm{eV}$, and $32 \mathrm{eV}$. The dashed lines are guides for the eye. The inset shows the comparison between the experimental data and simulations.
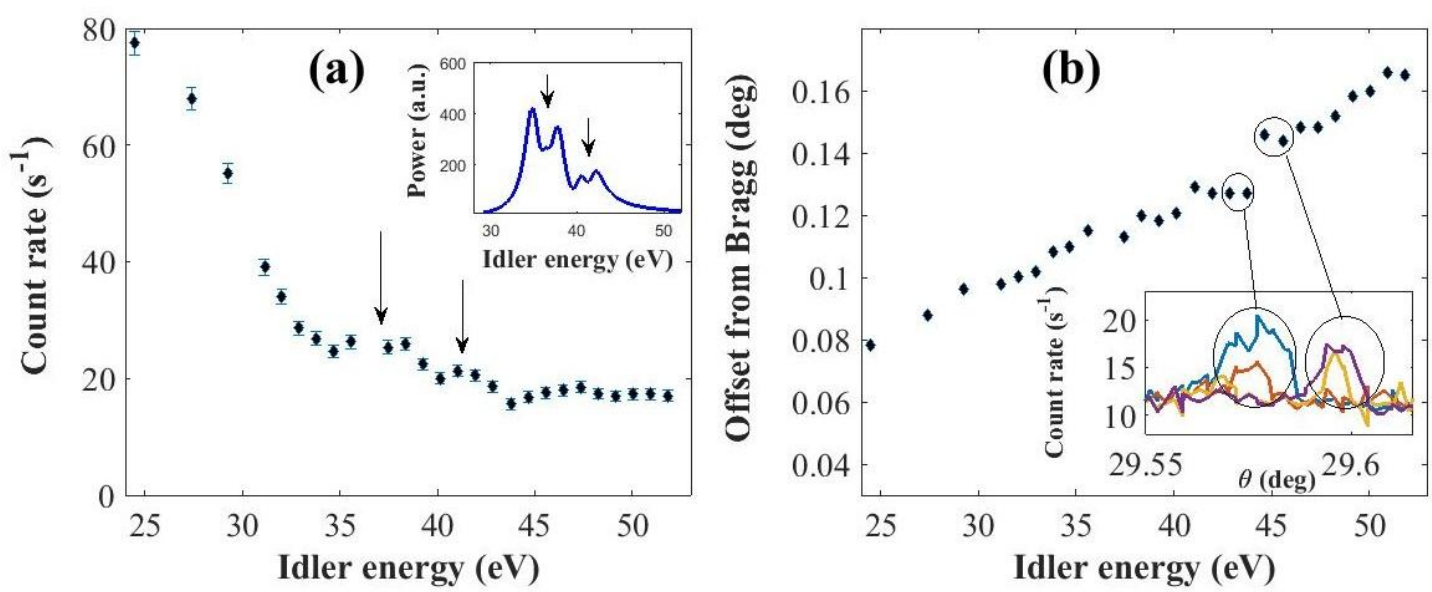

FIG. 4. (Color online) Experimental results showing the spectral dependencies on the idler photon energy of (a) the efficiency of the PDC and (b) the peak position of the signal beam. In (a) the arrows point at the photon energies at which the theory that includes the interaction with plasmons predicts peaks. The inset presents the simulation of the PDC process and the arrows point at energies that correspond to the 
position of the measured spectral peaks. In (b) the inset shows the rocking curves of the PDC at the photon energies near the sharp change in the peak position as a function of the idler photon energy. 